\title{
Endobronchial ultrasound-guided transbronchial needle aspiration in the diagnosis of lymphoma
}

\author{
M P Kennedy, ${ }^{1}$ C A Jimenez, ${ }^{1}$ J F Bruzzi, ${ }^{2}$ A D Mhatre, ${ }^{1}$ X Lei, ${ }^{3} \mathrm{~F}$ J Giles, ${ }^{4}$ T Fanning, ${ }^{5}$ \\ R C Morice, ${ }^{1}$ G A Eapen ${ }^{1}$
}

${ }^{1}$ Department of Pulmonary Medicine, University of Texas MD Anderson Cancer Center, Houston, Texas, USA;

${ }^{2}$ Department of Radiology,

University of Texas MD

Anderson Cancer Center

Houston, Texas, USA;

${ }^{3}$ Department of Statistics,

University of Texas MD

Anderson Cancer Center,

Houston, Texas, USA;

${ }^{4}$ Department of Leukemia,

University of Texas MD

Anderson Cancer Center,

Houston, Texas, USA

${ }^{5}$ Department of Pathology

University of Texas MD

Anderson Cancer Center.

Houston, Texas, USA

Correspondence to:

Dr G A Eapen, MD Anderson

Cancer Center, Department of

Pulmonary Medicine, Unit 403,

1515 Holcombe Boulevard,

Houston, Texas 77030, USA

geapen@mdanderson.org

Received 11 May 2007

Accepted 12 October 2007

Published Online First

26 October 2007

\begin{abstract}
Background: The diagnostic accuracy of endobronchial ultrasound-guided transbronchial needle aspiration (EBUSTBNA) for the diagnosis of lymphoma in patients with mediastinal lymphadenopathy is not well defined.

Methods: A retrospective review was performed of all patients with mediastinal lymphadenopathy referred for EBUS-TBNA between August 2005 and December 2006 in whom lymphoma was suspected based on prior history or clinical presentation. Mediastinal biopsy specimens were taken using a linear array ultrasonic bronchoscope (Olympus XBF-UC 160F) and a 22-gauge cytology needle (NA-202C Olympus) with on-site cytopathological support. The EBUS-TBNA result was compared with a reference standard of pathological tissue diagnosis or a composite of $\geqslant 6$ months of clinical follow-up with radiographic imaging.
\end{abstract}

Results: Of 236 patients who underwent EBUS-TBNA, 25 were eligible for inclusion. Indications for EBUS-TBNA were suspected mediastinal recurrence of lymphoma $(n=13)$ and mediastinal lymphadenopathy of unknown cause $(n=12)$. Adequate lymph node sampling was accomplished in 24/25 patients (96\%); there were no complications. EBUS-TBNA identified lymphoma in 10 patients and benign disease in 14 patients. There was one false negative EBUS-TBNA for lymphoma (lymphoma prevalence 11/25 (44\%)). Follow-up over a median of 10.5 months (range 1-19) confirmed stable or regressive lymphadenopathy in all 14 patients without a lymphoma diagnosis, consistent with a benign diagnosis. Overall, EBUS-TBNA had a sensitivity of $90.9 \%$, specificity of $100 \%$, positive predictive value of $100 \%$ and negative predictive value of $92.9 \%$ for the diagnosis of lymphoma. Conclusions: EBUS-TBNA is an accurate, safe and useful tool in the investigation of suspected lymphoma with isolated mediastinal adenopathy, and may diminish the need for more invasive procedures such as mediastinoscopy.

Lymphoma typically presents with lymphadenopathy in anatomical sites easily accessible to diagnostic biopsy or fine needle aspiration such as in the neck, axillae or inguinal regions. However, both Hodgkin's and non-Hodgkin's lymphoma may present with isolated mediastinal lymphadenopathy ${ }^{1}$ and, in such cases, mediastinoscopy or thoracotomy may be required to obtain diagnostic material with the attendant risk of morbidities related to anaesthesia, bleeding, infection and postprocedural pain.

Transbronchial needle aspiration (TBNA) is a minimally invasive safe technique that allows sampling of mediastinal nodes, often obviating the need for more invasive procedures such as mediastinoscopy. ${ }^{2}$ TBNA has expanded the role of bronchoscopy in the diagnosis of mediastinal pathology and lung cancer staging. ${ }^{3}$ However, the sensitivity of TBNA in the assessment of mediastinal lymphadenopathy varies from $15 \%$ to $85 \%$ and depends on the prevalence of malignant mediastinal lymphadenopathy in the study population, operator experience, needle used, lymph node site and number of aspirates. ${ }^{4-9}$ These limitations may explain why surveys show that only $10-30 \%$ of pulmonologists regularly use TBNA. ${ }^{10} 11$

By integrating ultrasound technology into a flexible bronchoscope, endobronchial ultrasound (EBUS) allows accurate definition of mediastinal structures and enhances site selection for transbronchial sampling. The first device available was a radial probe introduced through the working channel of a flexible bronchoscope prior to subsequent TBNA. This marginally improved the yield of TBNA, particularly in paratracheal lymph nodes. ${ }^{12}$ However, the recent development of an integrated linear array ultrasonic bronchoscope now allows for real time ultrasonic visualisation of the needle at the area of interest with a consequent improvement in diagnostic yield to $93.5 \%$ in mediastinal or hilar lymphadenopathy among a cancer predominant population. ${ }^{13}$ The usefulness of EBUS-TBNA in lung cancer staging has been reported with a sensitivity of $92-95 \%^{14-17}$ but, to our knowledge, there have been no studies of the use of either TBNA or EBUS-TBNA in patients with mediastinal adenopathy secondary to lymphoma. We therefore reviewed our clinical experience to examine the clinical use of EBUSTBNA in patients with mediastinal lymphadenopathy secondary to suspected lymphoma.

\section{METHODS \\ Patients}

Consecutive patients with mediastinal lymphadenopathy of unknown cause in whom lymphoma was suspected who were referred for EBUS-TBNA between August 2005 and December 2006 were reviewed. A suspicion of lymphoma was raised either because of a prior history of lymphoma, known lymphoma elsewhere, or because of a presentation compatible with new isolated mediastinal lymphoma based on a review of available clinical and radiographic data by the bronchoscopist performing the EBUS-TBNA procedure. An example of a compatible presentation was isolated idiopathic bulky mediastinal adenopathy with or without systemic symptoms. Patients were excluded if there was another likely cause for the lymphadenopathy suspected, such as lung cancer 
or other non-lymphoproliferative malignancy. Our institutional review board approved this analysis.

\section{Imaging evaluation prior to EBUS-TBNA}

Prior to EBUS-TBNA, patients underwent CT imaging of the thorax, with or without PET/CT scanning. PET/CT scanning was performed on an integrated scanner (Discovery ST-8, General Electric Medical Systems, Milwaukee, Wisconsin, USA) using 18-fluorodeoxyglucose (F-18 FDG). Quantification of FDG uptake within mediastinal lymph nodes was performed both visually and semi-quantitatively according to the following equation: maximum standardised uptake value (SUVmax) = mean measured activity within the volume of interest ( $\mathrm{mCi}$ / $\mathrm{ml}$ )/(injected dose of FDG (mCi)/body weight (g)). For each lymph node that was subsequently aspirated by EBUS-TBNA, the diameter of the short axis of the lymph node (in $\mathrm{mm}$ ) and the intensity of FDG uptake (SUVmax) were recorded by a diagnostic radiologist. A lymph node was considered to be FDGavid if the intensity of FDG uptake appeared greater than that of the normal surrounding mediastinal blood pool uptake (assessed visually) and if the SUVmax was $\geqslant 4$.

\section{EBUS-TBNA procedure}

All of the EBUS-TBNA procedures were performed by interventional pulmonology attendances $(n=3)$ with or without supervised fellows. Under general anaesthesia with ventilation via a laryngeal mask airway, standard conventional flexible bronchoscopy (model BF-T160 bronchoscope, Olympus, Japan) was first performed to examine the tracheobronchial tree. A linear array ultrasonic bronchscope (Olympus XBF-UC 160F) with dedicated 22-gauge needle (NA-202C, Olympus Ltd) was subsequently used to perform ultrasonic examination and transbronchial aspiration. The regional lymph node stations of the mediastinum and hilar regions were systematically imaged and measured (diameter of short axis) using the International staging system (Mountain classification). ${ }^{18}$ All visualised lymph nodes $>0.5 \mathrm{~cm}$ were sampled using real-time ultrasonic needle guidance. Doppler ultrasound was used to identify vessels as necessary. Each nodal station was sampled at least twice until

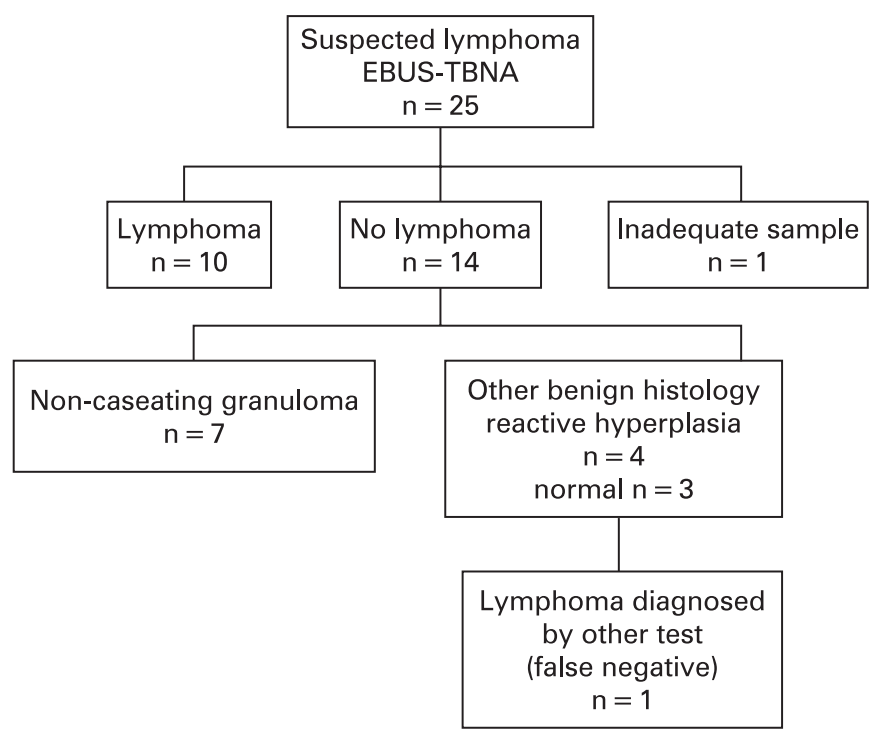

Figure 1 Patients suspected of having lymphoma undergoing endobronchial ultrasound-guided transbronchial needle aspiration (EBUSTBNA). adequate samples were attained by rapid on-site pathology evaluation (ROSE, or if an inadequate sample was initially obtained to a maximum of 5 passes per station). Specimen adequacy was defined by the presence of lymphoid tissue or tumour. Immunohistochemistry and flow cytometry were requested on all samples but actually performed on the samples at the discretion of the cytopathologist based on the cytomorphological characteristics. Adequate samples were defined as positive or negative for lymphoma. Negative samples were subdivided into benign disease, reactive hyperplasia or normal.

All complications were recorded and grouped into major complications (hospital admission, respiratory failure, bleeding requiring transfusion or other intervention, pneumothorax or pneumomediastinum) and minor complications (including postprocedure cough, sore throat and bleeding $<50 \mathrm{ml}$ controlled with simple bronchoscopic tamponade).

Reference standard evaluation: pathology and clinical follow-up For the purposes of the study, a true positive diagnosis of lymphoma was made if there was a positive biopsy of lymphoma within a mediastinal lymph node, either by EBUSTBNA or on subsequent follow-up by another modality within 6 months. A true negative diagnosis of benign disease was represented by a negative biopsy with stability or regression of lymphadenopathy documented on subsequent imaging (by CT and/or PET/CT) and by clinical follow-up over a period of at least 6 months. A diagnosis of sarcoidosis was made if EBUSTBNA or other tissue sample identified non-caseating granulomas along with a compatible clinical phenotype and adequate exclusion of other causes for granulomatous inflammation (clinical history, follow-up and a combination of negative tissue staining for acid-fast bacilli and fungal organisms and negative respiratory fungal and mycobacterial cultures).

\section{Statistical methods}

Summary statistics were used to report the lymph node characteristics. All the statistics analysis was performed in SAS Version 9.1 (SAS Institute, Cary, NC, USA).

\section{RESULTS}

Of 236 consecutive patients who underwent EBUS-TBNA during the study period, 25 patients with idiopathic mediastinal lymphadenopathy were eligible for inclusion in the study (table 1, fig 1). Thirteen of these patients had a previous history of lymphoma; the other 12 patients had no previous history of lymphoma but lymphoma was suspected based on radiography and clinical presentation.

Adequate lymphoid tissue was obtained by EBUS-TBNA in $24 / 25$ patients (96\%). Forty-nine lymph nodes were sampled in total (average 1.96 per patient; table 2). Based on the results of EBUS-TBNA, other investigations and clinical follow-up, 11 patients had a final diagnosis of mediastinal lymphoma. Followup over a median of 10.5 months (range 1-19) confirmed stable or regressive lymphadenopathy in all 14 patients $(n=8$ sarcoidosis) without a diagnosis of lymphoma, consistent with a benign diagnosis (table 1). Eight of 13 patients (61.5\%) with a prior diagnosis of lymphoma were finally diagnosed with lymphoma and EBUS-TBNA was diagnostic in all. Only 3/12 patients $(25 \%)$ without a prior history of lymphoma ended up with a final diagnosis of lymphoma and EBUS-TBNA was diagnostic in two and false negative in one other (described below). 
Table 1 Clinical characteristics, radiographic findings, endobronchial ultrasound transbronchial needle aspiration results and method of final diagnosis in 25 patients with mediastinal adenopathy with a suspicion of lymphoma

\begin{tabular}{|c|c|c|c|c|c|c|c|c|}
\hline \multirow[b]{2}{*}{$\begin{array}{l}\text { Patient no/ } \\
\text { sex/age }\end{array}$} & \multirow[b]{2}{*}{$\begin{array}{l}\text { Pre EBUS diagnosis } \\
\text { (years in remission) }\end{array}$} & \multicolumn{4}{|c|}{ Node characteristics } & \multirow[b]{2}{*}{$\begin{array}{l}\text { EBUS result (no of } \\
\text { stations) }\end{array}$} & \multirow[b]{2}{*}{ Follow-up } & \multirow[b]{2}{*}{$\begin{array}{l}\text { Final diagnosis by WHO } \\
\text { classification (method) }\end{array}$} \\
\hline & & Station & $\begin{array}{l}\text { EBUS size } \\
(\mathrm{mm})\end{array}$ & $\begin{array}{l}\text { CT size } \\
(\mathrm{mm})\end{array}$ & PET SUV & & & \\
\hline \multirow[t]{2}{*}{ 1/M/61 } & \multirow[t]{2}{*}{ NHL small cell lymphoma (1) } & 7 & 18 & 18 & 4.2 & \multirow[t]{2}{*}{ Lymphoma ( $\mathrm{n}=2$ ) } & \multirow[t]{2}{*}{ Lymphoma treatment } & \multirow{2}{*}{$\begin{array}{l}\text { Small lymphocytic } \\
\text { lymphoma }(C, I, F I)\end{array}$} \\
\hline & & $11 R$ & 18 & 25 & 5.9 & & & \\
\hline 2/M/64 & NHL small cell lymphoma (3) & $4 \mathrm{~L}$ & 14 & 21 & $\mathrm{n} / \mathrm{p}$ & Lymphoma & Lymphoma treatment & $\begin{array}{l}\text { Small lymphocytic } \\
\text { lymphoma }(C, I, F I)\end{array}$ \\
\hline \multirow[t]{2}{*}{$3 / F / 70$} & \multirow[t]{2}{*}{ NHL small cell lymphoma (3) } & 7 & 9 & 9 & 5.1 & \multirow[t]{2}{*}{ Lymphoma ( $\mathrm{n}=2$ ) } & \multirow[t]{2}{*}{ Lymphoma treatment } & \multirow{2}{*}{$\begin{array}{l}\text { Small lymphocytic } \\
\text { lymphoma }(C, I, F I)\end{array}$} \\
\hline & & $11 R$ & 10 & 16 & 7.4 & & & \\
\hline \multirow[t]{2}{*}{$4 / F / 75$} & \multirow[t]{2}{*}{ Discordant NHL (4) } & $4 \mathrm{~L}$ & 7 & 9 & 3.9 & 4L: Normal & \multirow[t]{2}{*}{ Lymphoma treatment } & Follicular lymphoma* \\
\hline & & $10 \mathrm{R}$ & 12 & 13 & 4.4 & 10R Lymphoma & & \\
\hline $5 / F / 63$ & NHL large cell lymphoma (1) & 7 & 14 & 13 & 8.9 & 7: Lymphoma & Lymphoma treatment & Large cell lymphoma $(\mathrm{C}, \mathrm{I})$ \\
\hline $6 / F / 61$ & NHL large cell s/p BMT (1) & 7 & 9 & 14 & 18.2 & 7: Lymphoma & Lymphoma treatment & $\begin{array}{l}\text { Large cell lymphoma** } \\
(\mathrm{C}, \mathrm{I})\end{array}$ \\
\hline 7/M/67 & Mantle cell lymphoma (1) & $4 \mathrm{R}$ & 11 & 13 & $\mathrm{n} / \mathrm{p}$ & 4R: Lymphoma & Lymphoma treatment & Mantle cell lymphoma \\
\hline & & $11 R$ & 19 & 16 & $\mathrm{n} / \mathrm{p}$ & 11R: Lymphoma & & $(\mathrm{C}, \mathrm{I}, \mathrm{FI})$ \\
\hline $8 / F / 34$ & Hodgkin's lymphoma (1) & 7 & 13 & 17 & 7.9 & 7: Hodgkin's & Lymphoma treatment & Hodgkin's lymphoma $(\mathrm{C}, \mathrm{I})$ \\
\hline & & $11 R$ & 12 & 18 & 15.6 & 11R: Normal & & \\
\hline $9 / \mathrm{M} / 49$ & Hodgkin's lymphoma (7) & 7 & 14 & 12 & 20.7 & 7: Normal & 12 months: PET/CT resolution & Sarcoid-like \\
\hline & & $11 \mathrm{R}$ & 14 & 10 & 13.7 & 11R: Granuloma & of lymphadenopathy & lymphadenopathy \\
\hline $10 / \mathrm{M} / 65$ & NHL small cell lymphoma (6) & $11 R$ & 10 & 11 & 4.8 & Reactive hyperplasia & $\begin{array}{l}11 \text { months: PET/CT resolution } \\
\text { of lymphadenopathy }\end{array}$ & Benign lymphadenopathy \\
\hline $11 / \mathrm{M} / 70$ & NHL small cell lymphoma (1) & $4 \mathrm{R}$ & 7 & 4 & 1.8 & Reactive hyperplasia & 9 months: CT resolution & Pseudomonas pneumonia \\
\hline & & $4 \mathrm{~L}$ & 7 & 8 & 2.5 & $(n=4)$ & of lymphadenopathy & \\
\hline & & 7 & 13 & 7 & 2.5 & & & \\
\hline & & $11 R$ & 8 & 11 & 3 & & & \\
\hline $12 / F / 48$ & Hodgkin's lymphoma s/p BMT & $4 \mathrm{R}$ & 8 & 18 & 3.7 & No lymphocytes & 12 months: CT resolution of & Drug-induced pneumonitis \\
\hline & & 7 & 8 & 21 & 1.1 & $(n=2)$ & lymphadenopathy & \\
\hline 13/F/73 & Mycosis fungoides & $4 \mathrm{R}$ & 10 & 19 & 4.5 & Reactive hyperplasia & 16 months: CT no & Benign lymphadenopathy \\
\hline & & $4 \mathrm{~L}$ & 10 & 10 & 3.9 & $(n=3)$ & change & \\
\hline & & 7 & 14 & 9 & 4 & & & \\
\hline 14/M49 & ?Lymphoma & 7 & 20 & 40 & 21.2 & Hodgkin's lymphoma & Lymphoma treatment & Hodgkin's lymphoma† \\
\hline & & $11 \mathrm{R}$ & 27 & 30 & 20.6 & $(n=2)$ & & $(\mathrm{C}, \mathrm{I}, \mathrm{H})$ \\
\hline $15 / F / 27$ & ?Lymphoma & $11 R$ & 7 & 14 & $\mathrm{n} / \mathrm{p}$ & 11R: Lymphoma & Lymphoma treatment & $\begin{array}{l}\text { Hodgkin's lymphoma†† } \\
(\mathrm{C}, \mathrm{I}, \mathrm{H})\end{array}$ \\
\hline $16 / \mathrm{M} / 70$ & ?Lymphoma & 7 & 13 & 16 & $\mathrm{n} / \mathrm{p}$ & Normal & $\begin{array}{l}8 \text { months: } \$ \text { CT reduction } \\
\text { in size }\end{array}$ & Follicular lymphoma§ (C,I) \\
\hline $17 / \mathrm{M} / 65$ & ?Lymphoma & $11 \mathrm{R}$ & 10 & 9 & 3.6 & Normal & 10 months: PET/CT no change & $\begin{array}{l}\text { Lymphoproliferative } \\
\text { disordertt }\end{array}$ \\
\hline $18 / F / 69$ & ?Lymphoma & $4 \mathrm{R}$ & 17 & 13 & 6.4 & Reactive hyperplasia & 7 months: PET/CT & Chronic eosinophilic \\
\hline & & $4 \mathrm{~L}$ & 8 & 14 & 7.9 & $(n=2)$ & reduction in SUV and size & Pneumonia \\
\hline $19 / \mathrm{M} / 44$ & ?Lymphoma & $4 \mathrm{R}$ & 10 & 11 & $\mathrm{n} / \mathrm{p}$ & Granuloma $(n=2)$ & 19 months: CT stable & Sarcoidosis \\
\hline & & 7 & 10 & 8 & $\mathrm{n} / \mathrm{p}$ & & & \\
\hline $20 / F / 48$ & ?Lymphoma & $4 \mathrm{R}$ & 10 & 15 & $\mathrm{n} / \mathrm{p}$ & Normal $(n=2)$ & 9 months: CT stable & Sarcoidosis \\
\hline & & 7 & 9 & 12 & $\mathrm{n} / \mathrm{p}$ & & & \\
\hline $21 / F / 56$ & ?Lymphoma & $4 \mathrm{R}$ & 17 & 17 & $\mathrm{n} / \mathrm{p}$ & Granuloma $(n=2)$ & 18 months: CT reduction & Sarcoidosis $\uparrow$ \\
\hline & & 7 & 21 & 19 & $\mathrm{n} / \mathrm{p}$ & & in size & \\
\hline $22 / F / 59$ & ?Lymphoma & $4 \mathrm{~L}$ & 15 & 16 & 9.2 & 4L: Granuloma & 1 month: CT stable & Sarcoidosis $\uparrow$ \\
\hline & & 7 & 12 & 12 & 6.5 & 7: Normal & & \\
\hline & & $11 R$ & 9 & 10 & 7.9 & 11R: Inadequate & & \\
\hline 23/M/77 & ?Lymphoma & $4 \mathrm{R}$ & 12 & 15 & $\mathrm{n} / \mathrm{p}$ & 4R: Normal & 15 months: CT reduction & Sarcoidosis \\
\hline & & 7 & 11 & 7 & $\mathrm{n} / \mathrm{p}$ & 7: Granuloma & in size & \\
\hline & & $11 \mathrm{R}$ & 13 & 10 & $\mathrm{n} / \mathrm{p}$ & 11R: Normal & & \\
\hline $24 / \mathrm{M} / 42$ & ?Lymphoma & $3 \mathrm{P}$ & 19 & 18 & $\mathrm{n} / \mathrm{p}$ & Granuloma $(\mathrm{n}=3)$ & 4 months: CT reduction & Sarcoidosis \\
\hline & & $4 \mathrm{R}$ & 13 & 22 & $\mathrm{n} / \mathrm{p}$ & & in size & \\
\hline & & 7 & 13 & 15 & $\mathrm{n} / \mathrm{p}$ & & & \\
\hline $25 / F / 51$ & ?Lymphoma & $4 \mathrm{R}$ & 7 & 15 & $\mathrm{n} / \mathrm{p}$ & Granuloma $(\mathrm{n}=2)$ & 10 months: CT reduction & Sarcoidosis \\
\hline & & 7 & 16 & 18 & $\mathrm{n} / \mathrm{p}$ & & in size & \\
\hline
\end{tabular}

BMT, bone marrow transplant; C, cytology; EBUS, endobronchial ultrasound; Fl, flow cytometry; H, histology; I, immunohistochemistry; N/A, not applicable, NHL, non-Hodgkin's lymphoma; NSCLC, non-small cell lung cancer; n/p, not performed; S/P status post; Max PET SUV, maximum positron emission tomography standardised uptake value.

${ }^{*}$ Atypical cells consistent with follicular lymphoma confirmed by flow cytometry and immunohistochemistry from supraclavicular lymph node aspiration. $†$ Confirmed by mediastinoscopy. \$Despite no lymphoma therapy. §Diagnosed by CT-guided mesenteric lymph node biopsy. $\uparrow$ Granuloma identified by other diagnostic test. **Repeat CT 6 weeks later identified a new posterior mediastinal mass which also positive for lymphoma by CT-guided fine needle aspiration. † †one marrow and endobronchial biopsies also positive for Hodgkin's lymphoma. t. Oral Epstein-Barr virus-associated lymphoproliferative lesion (spontaneous resolution). 
Of the 11 patients with a final diagnosis of lymphoma, EBUSTBNA correctly diagnosed lymphoma in 10. There was one false negative case (described below). Of the 10 patients diagnosed with lymphoma by EBUS-TBNA, 3 patients were diagnosed with Hodgkin's lymphoma independently by EBUS-TBNA, 1 of whom had a previous history of Hodgkin's lymphoma (patient 8). One patient with Hodgkin's lymphoma was initially diagnosed on EBUS-TBNA based on the cytological finding of Reed-Sternberg cells (patient 14, fig 2), but went on to undergo a medistinoscopy for further subclassification with an ultimate diagnosis of Hodgkin's lymphoma, nodular sclerosing type. The other patient with Hodgkin's lymphoma (patient 15) was diagnosed definitively by EBUS-TBNA and confirmed by bone marrow and endobronchial biopsies. The other 8 patients had a final diagnosis of non-Hodgkin's lymphoma. The method of diagnosis of lymphoma by EBUS-TBNA was a combination of cytology, immunohistochemistry with and without flow cytometry in 6 and 4 patients, respectively $(2$ also had histological confirmation by biopsy (mediastinoscopy and endobronchial biopsy); table 1).

In the 14 patients without lymphoma, EBUS correctly diagnosed benign disease (non-caseating granuloma consistent with sarcoidosis) in 8 patients, reactive hyperplasia or normal lymphoid tissue in 5 and an inadequate sample was obtained in 1 patient (described below). The diagnostic accuracy of EBUSTBNA in patients with suspected lymphoma was 24/25 (96\%) and the sensitivity, specificity, positive and negative predictive values were $90.9 \%, 100 \%, 100 \%$ and $92.6 \%$, respectively (table 3).

\section{Performance of EBUS-TBNA according to clinical presentation: patients with a prior history of lymphoma $(n=13)$}

Of the 25 patients who underwent EBUS-TBNA for a suspicion of lymphoma, 13 had a prior history of lymphoma. Eleven were in remission, one had an autologous bone marrow transplant 4 months previously and one had active mycosis fungoides. The mean period of remission in the 11 patients was 2.6 years and they were referred for EBUS-TBNA for new or recurrent mediastinal lymph node enlargement. EBUS-TBNA diagnosed lymphoma recurrence in 8 of these 11 patients; there was no evidence of progressive lymphadenopathy on follow-up of the

Table 2 Summary statistics for lymph node size $(\mathrm{mm})$ by EBUS and CT and PET standardised uptake values in 25 patients with mediastinal lymphadenopathy who underwent EBUS-guided transbronchial biopsy for a suspicion of lymphoma

\begin{tabular}{lll}
\hline Variables & EBUS positive & EBUS negative \\
\hline CT size (mm) & & \\
$N$ & 14 & 35 \\
Mean (SD) & $18.50(8.23)$ & $12.86(4.22)$ \\
Median & 16.0 & 12.0 \\
$\quad$ Range & $9-40$ & $4-22$ \\
Ultrasound size (mm) & & \\
N & 14 & 35 \\
Mean (SD) & $14.36(5.47)$ & $11.54(3.62)$ \\
Median & 13.5 & 11.0 \\
Range & $7-27$ & $7-21$ \\
PET SUV & & \\
N & 10 & 20 \\
Mean (SD) & $10.38(6.85)$ & $6.36(5.06)$ \\
Median & 7.65 & 4.25 \\
Range & $4.2-21.2$ & $1.1-20.7$ \\
\hline
\end{tabular}

EBUS, endobronchial ultrasound; PET, positron emission tomography; SUV, standardised uptake value. other 3 patients and the EBUS-TBNA findings were considered true negative.

One patient with a history of Hodgkin's lymphoma who had received an autologous bone marrow transplant with conditioning chemotherapy (melphalan and busulphan) 4 months earlier presented with a cough and pulmonary infiltrates. The tissue sample obtained by EBUS-TBNA was inadequate owing to a dense fibrous capsule surrounding the lymph nodes at mediastinal stations 7 and $4 \mathrm{R}$ that resisted penetration with the cytology needle. A presumptive diagnosis of busulphan-induced pneumonitis was made with response to steroid treatment and stable lympadenopathy on CT scanning 8 months later. The other patient was undergoing treatment for mycosis fungoides and EBUS-TBNA identified reactive lymph node hyperplasia consistent with clinical and radiological follow-up.

\section{Performance of EBUS-TBNA according to clinical presentation: patients with no prior history of lymphoma $(n=12)$}

Twelve of the patients in the study group presented with mediastinal lymphadenopathy with no prior history of lymphoma but in whom lymphoma was suspected. EBUS-TBNA revealed the diagnosis of Hodgkin's lymphoma in 2 patients, benign lymph node hyperplasia in 3 (transbronchial biopsies were positive for non-caseating granuloma in 1 patient) and non-caseating granuloma consistent with sarcoidosis in 6 patients.

One other patient presented with right flank pain and both mediastinal and mesenteric lymphadenopathy. Both EBUSTBNA and CT-guided biopsy of the mesenteric adenopathy were performed: although the CT-guided biopsy diagnosed mesenteric non-Hodgkin's B cell lymphoma (largest node: $18 \mathrm{~mm}$ short axis diameter), EBUS-TBNA revealed normal lymphoid tissue in the mediastinum (subcarinal lymph node station 7: $16 \mathrm{~mm}$ short axis diameter). Two subsequent PET scans showed no FDG-avid disease in the mediastinum. The patient declined systemic chemotherapy; at 8 months of followup there had been a progressive increase in size in the mesenteric lymphadenopathy but, interestingly, the mediastinal lymphadenopathy had reduced in size. Despite this clear discrepancy in the clinical course of the mediastinal adenopathy, for purposes of this analysis we considered the EBUS-TBNA result as a false negative for mediastinal lymphoma.

\section{Complications}

No complications were identified from the procedure or anaesthesia. All procedures were performed as outpatient cases and all patients were discharged after brief observation as per institutional protocol.

\section{DISCUSSION}

Our findings show that EBUS-TBNA is a safe and diagnostically accurate technique for the diagnosis of lymphoma in patients with mediastinal lymphadenopathy.

Obtaining adequate pathological specimens is vital in the definitive diagnosis of lymphoma. In patients with mediastinal adenopathy - including those with suspected isolated mediastinal lymphoma-mediastinoscopy has been the procedure of choice to obtain tissue over the past three decades. ${ }^{19}$ However, mediastinoscopy does not allow access to perihilar lymph nodes and it is technically very difficult to repeat the mediastinoscopy on the same patient due to post-procedural fibrosis. This becomes particularly important in previously treated lymphoma patients with recurrent mediastinal adenopathy. It is also a 

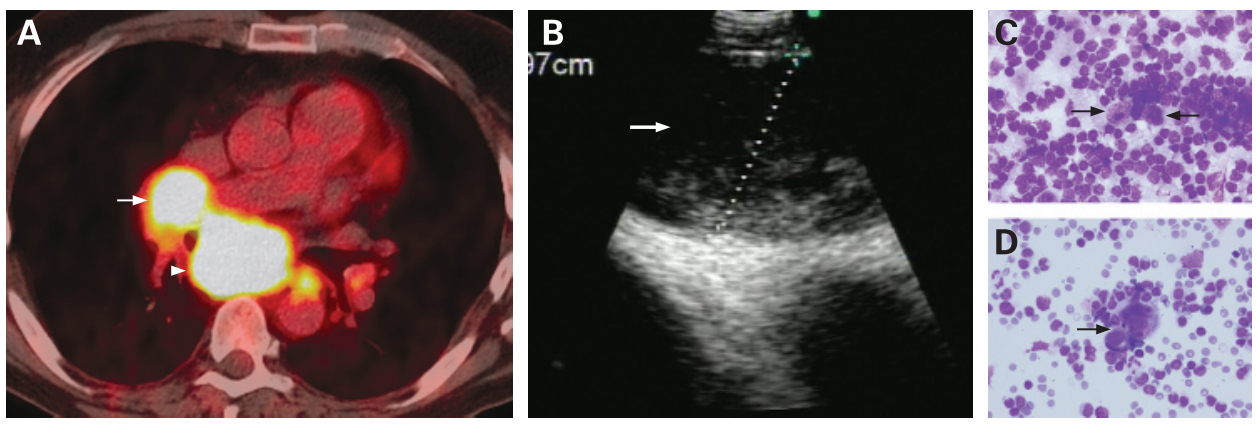

Figure 2 Radiographic, endobronchial ultrasound (EBUS) and pathological images of a 49-year-old man with no significant past medical history who underwent endobronchial ultrasound transbronchial needle aspiration (EBUS-TBNA) for the investigation of idiopathic mediastinal lymphadenopathy. (A) Axial fused PET/CT image showing intense uptake of fluorodeoxyglucose (FDG) within the enlarged lymph nodes (stations 7 (arrow head) and 11R (arrow)), suspicious for malignancy. (B) EBUS image showing an enlarged station 7 lymph node (arrow). (C, D) Fine needle aspirate by EBUS-TBNA showing atypical lymphoid population favouring Hodgkin's lymphoma with two Reed-Sternberg variants (C, arrows) and a multinucleated "lacunar variant" Reed-Sternberg cell (D, arrow). Stain, Diff-Quik; magnification, $400 \times$.

surgical procedure with attendant risks and complications. In several large series of patients with mediastinal adenopathy undergoing lung cancer staging, definitive diagnosis by mediastinoscopy was achieved in approximately $85-95 \%$ of cases with false negatives rates of 5-8\%. ${ }^{19-21}$ Significant complications occurred in $1-5 \% .^{19-21}$ Thus, newer less invasive methods of obtaining pathological specimens have been developed including CT-guided transthoracic needle biopsy and transbronchial and transoesophageal lymph node sampling.

The yield of TBNA in patients with lymphoma has not been well defined. We reviewed the literature and identified a number of series reporting the yield of TBNA in mediastinal adenopathy using a variety of cytology (21-22 gauge) and histology needles (19 gauge). ${ }^{5-8}$ These reports included 8 patients with mediastinal adenopathy secondary to lymphoma in whom TBNA missed the diagnosis in over $60 \%{ }^{5-7}$

In contrast, the performance of endoscopic ultrasound-guided fine needle aspiration (EUS-FNA) (22 gauge needle) in one study of 23 patients in lymphoma was reported with sensitivity, specificity and accuracy of $74 \%, 93 \%$ and $81 \% .{ }^{22}$ These authors also asserted that the addition of flow cytometry and immunohistochemistry improved the yield of EUS-FNA. A more recent study also suggested that the addition of histological sampling using a 19-gauge needle to EUS-FNA in patients with mediastinal adenopathy without ROSE improved the diagnosis of lymphoma. ${ }^{23}$ The diagnostic reach of EUS-FNA is, however, limited to retrotracheal (station 3), left lower paratracheal (station 4L), subcarinal (station 7), lower paraoesophageal (station 8) and pulmonary ligament (station 9) lymph nodes.

Table 3 Performance of endobronchial ultrasound transbronchial needle aspiration (EBUS-TBNA) in a population of 25 patients with suspected lymphoma

\begin{tabular}{lcc}
\hline & Lymphoma present & Lymphoma absent \\
\hline EBUS-TBNA positive & 10 & 0 \\
EBUS-TBNA negative & 1 & 13 \\
Adequate lymphoid tissue: $24 / 25(96 \%)^{*}$ & \\
Sensitivity $90.9 \%(95 \% \mathrm{Cl} 0.74$ to 1.00$)$ \\
Specificity $100 \%(95 \% \mathrm{Cl} 0.75$ to 1.00$)$ \\
Positive predictive value $100 \%(95 \% \mathrm{Cl} 0.69$ to 1.00$)$ \\
Negative predictive value $92.9 \%(95 \% \mathrm{Cl} 0.79$ to 1.00$)$
\end{tabular}

*One patient with an inadequate sample was excluded from the $2 \times 2$ table.
EBUS-TBNA, on the other hand, allows access to the upper and lower paratracheal (stations 1, 2, 3P and 4), subcarinal (station 7), peribronchial and perihilar (stations 10, 11 and 12) lymph nodes. ${ }^{17}$ Indeed, EUS-FNA and EBUS-TBNA appear to be complementary and combining both approaches has been shown to improve the diagnostic yield in patients with mediastinal adenopathy. ${ }^{24}$

Although this report is limited by retrospection and size, as far as we are aware it is the first published analysis of the diagnostic usefulness of EBUS-TBNA in patients with mediastinal lymphadenopathy suspected of being due to lymphoma. The sensitivity, specificity and accuracy of EBUS-TBNA in our patients with mediastinal lymphoma were comparable to previously reported experience with EUS-FNA. ${ }^{24}$ In fact, it could be argued that the one false negative result we identified by EBUS-TBNA (patient 16) may represent a true negative result since the patient's mediastinal lymphadenopathy decreased in size in the absence of any chemotherapy. Lymphoma was diagnosed by EBUS-TBNA more often in patients with a previous history of lymphoma, but this appears to be related more to the prevalence of the disease in the subgroup than any limitation attributable to the technique. Overall, the diagnostic yield of EBUS-TBNA for mediastinal lymphoma was substantially better than the historical data for conventional TBNA, particularly for non-Hodgkin's lymphoma, and may even be comparable to the $90 \%$ sensitivity reported for mediastinoscopy. ${ }^{25}$

Since the commencement of our EBUS programme we have routinely used flow cytometry and immunohistochemistry in patients with suspected lymphoma on the basis of previously published experience with EUS-FNA, and our data suggest that this is a reasonable clinical approach. ${ }^{22}$ Unlike EUS-FNA, a 19gauge trucut biopsy needle is not currently available for realtime EBUS-TBNA and it is plausible that this additional histological sampling will improve the yield and sensitivity of EBUS-TBNA in patients with mediastinal adenopathy secondary to lymphoma.

In conclusion, in patients with mediastinal adenopathy and a suspicion of lymphoma, EBUS-TBNA with flow cytometry and immunohistochemistry analysis is a safe, minimally invasive and highly accurate technique that may decrease the need for more invasive procedures such as mediastinoscopy.

Competing interests: None 


\section{REFERENCES}

1. Duwe BV, Sterman DH, Musani Al. Tumors of the mediastinum. Chest 2005:128:2893-909.

2. Wang KP, Terry P, Marsh B. Bronchoscopic needle aspiration biopsy of paratracheal tumors. Am Rev Respir Dis 1978;118:17-21.

3. Herth FJ, Rabe KF, Gasparini S, et al. Transbronchial and transoesophageal (ultrasound-guided) needle aspirations for the analysis of mediastinal lesions. Eur Respir J 2006;28:1264-75.

4. Holty JE, Kuschner WG, Gould MK. Accuracy of transbronchial needle aspiration for mediastinal staging of non-small cell lung cancer: a meta-analysis. Thorax 2005;60:890-1.

5. Sharafkhaneh A, Baaklini W, Gorin AB, et al. Yield of transbronchial needle aspiration in diagnosis of mediastinal lesions. Chest 2003;124:2131-5.

6. Cetinkaya E, Yildiz P, Altin S, et al. Diagnostic value of transbronchial needle aspiration by Wang 22-gauge cytology needle in intrathoracic lymphadenopathy. Chest 2004;125:527-31

7. Selcuk ZT, Firat P. The diagnostic yield of transbronchial needle aspiration in superior vena cava syndrome. Lung Cancer 2003;42:183-8.

8. Hermens FH, Van Engelenburg TC, Visser FJ, et al. Diagnostic yield of transbronchial histology needle aspiration in patients with mediastinal lymph node enlargement. Respiration 2003:70:631-5.

9. Patel NM, Pohlman A, Husain A, et al. Conventional transbronchial needle aspiration decreases the rate of surgical sampling of intrathoracic lymphadenopathy. Chest 2007;131:773-8.

10. Haponik EF, Shure D. Underutilization of transbronchial needle aspiration: experiences of current pulmonary fellows. Chest 1997:112:251-3.

11. Smyth CM, Stead RJ. Survey of flexible fibreoptic bronchoscopy in the United Kingdom. Eur Respir J 2002;19:458-63.

12. Herth F, Becker HD, Ernst A. Conventional vs endobronchial ultrasound-guided transbronchial needle aspiration: a randomized trial. Chest 2004;125:322-5.

13. Herth FJ, Eberhardt R, Vilmann $P$, et al. Real-time endobronchial ultrasound guided transbronchial needle aspiration for sampling mediastinal lymph nodes. Thorax 2006;61:795-8.
14. Yasufuku K, Chiyo M, Sekine $Y$, et al. Real-time endobronchial ultrasound-guided transbronchial needle aspiration of mediastinal and hilar lymph nodes. Chest 2004;126:122-8

15. Yasufuku K, Chiyo M, Koh E, et al. Endobronchial ultrasound guided transbronchial needle aspiration for staging of lung cancer. Lung Cancer 2005:50:347-54.

16. Herth FJ, Ernst A, Eberhardt R, et al. Endobronchial ultrasound-guided transbronchial needle aspiration of lymph nodes in the radiologically normal mediastinum. Eur Respir J 2006;28:910-4.

17. Yasufuku K, Nakajima T, Motoori K, et al. Comparison of endobronchial ultrasound positron emission tomography, and CT for lymph node staging of lung cancer. Chest 2006:130:710-8.

18. Mountain CF, Dresler CM. Regional lymph node classification for lung cancer staging. Chest 1997;111:1718-23.

19. Hujala KT, Sipila JI, Grenman R. Mediastinoscopy: its role and value today in the differential diagnosis of mediastinal pathology. Acta Oncol 2001:40:79-82.

20. Hammoud ZT, Anderson RC, Meyers BF, et al. The current role of mediastinoscopy in the evaluation of thoracic disease. J Thorac Cardiovasc Surg 1999;118:894-9.

21. Lemaire A, Nikolic I, Petersen T, et al. Nine-year single center experience with cervical mediastinoscopy: complications and false negative rate. Ann Thorac Surg 2006;82:1185-9

22. Ribeiro A, Vazquez-Sequeiros E, Wiersema LM, et al. EUS-guided fine-needle aspiration combined with flow cytometry and immunocytochemistry in the diagnosis of lymphoma. Gastrointest Endosc 2001;53:485-91.

23. Storch I, Jorda M, Thurer R, et al. Advantage of EUS Trucut biopsy combined with fine-needle aspiration without immediate on-site cytopathologic examination. Gastrointest Endosc 2006;64:505-11.

24. Herth FJ, Lunn W, Eberhardt R, et al. Transbronchial versus transesophageal ultrasound-guided aspiration of enlarged mediastinal lymph nodes. Am J Respir Crit Care Med 2005;171:1164-7.

25. Porte H, Roumilhac D, Eraldi L, et al. The role of mediastinoscopy in the diagnosis of mediastinal lymphadenopathy. Eur J Cardiothorac Surg 1998;13:196-9.

\section{Lung alert}

\section{A new biomarker in asthma?}

This is the first study to evaluate the pathogenic role of the novel chitinase-like protein YKL-40 in asthma. In experimental models, YKL-40 and chitinases promoted airway inflammation through type 2 helper T cells. This study investigated serum YKL-40 levels in asthma, including assessing relationships with disease severity, airway YKL-40 levels and airway remodelling.

Serum YKL-40 levels, measured by immunoassay, were investigated in a prospective casecontrol study involving three separate cohorts of patients with asthma from Yale, Paris and Wisconsin. Serum YKL-40 levels were significantly raised in patients with asthma compared with controls. They correlated with asthma severity assessed by clinical and lung function variables. This significant relationship persisted after adjusting for age, sex, ethnicity and body mass index. It was predominantly due to high serum YKL-40 levels in the subpopulation with severe asthma. Bronchoscopy, only undertaken in the cohort from Paris, showed a positive relationship between serum and airway YKL-40 levels and subepithelial basement membrane thickness.

These findings suggest that YKL-40 has pathogenic importance in some patients with severe asthma, and represents a potential novel therapeutic target. Further research is needed to confirm this because the present study has some limitations. The patient cohorts were relatively small with different inclusion and exclusion criteria. Patients did not receive standard treatments. As the study was cross-sectional, changes over time were not evaluated. The effects of asthma exacerbations were not examined and bronchoscopic data were restricted to one cohort.

- Chupp GL, Lee CG, Jarjour N, et al. A chitinase-like protein in the lung and circulation of patients with severe asthma. N Engl J Med 2007; 357:2016-27

\section{Ian Scott}

Correspondence to: I Scott, ST2, Royal Albert Edward Infirmary, Wigan, UK; ian.c.scott@btinternet.com 